\title{
Revised SED of the triple protostellar system VLA 1623-2417
}

\author{
N. M. Murillo ${ }^{1}$, D. Harsono ${ }^{1}$, M. McClure ${ }^{2}$, S.-P. Lai ${ }^{3,4}$, and M. R. Hogerheijde ${ }^{1,2}$ \\ ${ }^{1}$ Leiden Observatory, Leiden University, P.O. Box 9513, 2300 RA, Leiden, The Netherlands \\ e-mail: nmurillo@strw. leidenuniv.nl \\ 2 Anton Pannekoek Institute for Astronomy, University of Amsterdam, Science Park 904, 1098 XH, Amsterdam, The Netherlands \\ 3 Institute of Astronomy and Department of Physics, National Tsing Hua University, 101 Section 2 Kuang Fu Road, Hsinchu 30013, \\ Taiwan \\ 4 Academia Sinica Institute of Astronomy and Astrophysics, P.O. Box 23-141, 10617 Taipei, Taiwan
}

Received 14 May 2018 / Accepted 2 July 2018

\begin{abstract}
Context. VLA 1623-2417 is a triple protostellar system deeply embedded in Ophiuchus A. Sources A and B have a separation of 1.1", making their study difficult beyond the submillimeter regime. Lack of circumstellar gas emission suggested that VLA 1623-2417 B has a very cold envelope and is much younger than source A, which is generally considered the prototypical Class 0 source.

Aims. We explore the consequences of new ALMA Band 9 data on the spectral energy distribution (SED) of VLA 1623-2417 and their inferred nature.

Methods. We constructed and analyzed the SED of each component in VLA 1623-2417 using dust continuum observations spanning from centimeter to near-infrared wavelengths.

Results. The ALMA Band 9 data presented in this work show that the SED of VLA 1623-2417 B does not peak at $850 \mu \mathrm{m}$ as previously expected, but instead presents the same shape as VLA 1623-2417 A at wavelengths shorter than $450 \mu \mathrm{m}$.

Conclusions. The results presented in this work indicate that the previous assumption that the flux in Herschel and Spitzer observations is solely dominated by VLA 1623-2417 A is not valid, and instead, VLA 1623-2417 B most likely contributes a significant portion of the flux at $\lambda<450 \mu \mathrm{m}$. These results, however, do not explain the lack of circumstellar gas emission and puzzling nature of VLA $1623-2417$ B.
\end{abstract}

Key words. stars: formation - stars: low-mass - ISM: individual objects: VLA 1623-2417 - methods: observational techniques: interferometric

\section{Introduction}

VLA 1623-2417 (hereafter VLA 1623) is a deeply embedded protostellar system in Ophiuchus A (L1688) that was first observed by André et al. (1990). Recent observations place L1688 and VLA 1623 at a distance of $137.3 \pm 1.2$ pc (Ortiz-León et al. 2017). VLA 1623 is generally considered to be the prototypical Class 0 source (André et al. 1993). Observations at centimeter wavelengths with the Very Large Array (VLA) revealed several continuum peaks (Bontemps \& Andre 1997), which were interpreted as the central object of VLA 1623 and jet knots A, B, and C. However, observations of the outflow suggested more than one component is present in VLA 1623 (Dent et al. 1995; Yu \& Chernin 1997; Caratti o Garatti et al. 2006). Interferometric observations in submillimeter wavelengths revealed that VLA 1623 is actually a triple protostellar system (Looney et al 2000; Murillo \& Lai 2013), and each component drives an outflow of its own (Murillo \& Lai 2013; Santangelo et al. 2015). The VLA interferometric centimeter peaks A and B matched with sources B and W, respectively (Ward-Thompson et al. 2011; Murillo \& Lai 2013). No submillimeter counterpart was observed for the centimeter peak $\mathrm{C}$ detected with the VLA.

VLA $1623 \mathrm{~A}$ is brightest in the submillimeter wavelength range, presenting a centrally peaked source with extended dust emission coinciding with the Keplerian disk traced in $\mathrm{C}^{18} \mathrm{O}$
(Murillo et al. 2013). VLA 1623 B is separated from source A by $1.1^{\prime \prime}$ to the west and presents a strongly collimated outflow (Santangelo et al. 2015). Interferometric observations do not detect warm $\left(\mathrm{C}^{18} \mathrm{O}, \mathrm{c}-\mathrm{C}_{3} \mathrm{H}_{2}\right)$ or cold $\left(\mathrm{DCO}^{+}, \mathrm{N}_{2} \mathrm{D}^{+}, \mathrm{N}_{2} \mathrm{H}^{+}\right)$ gas emission associated with VLA 1623 B (Murillo et al. 2013, 2016, 2018). Submillimeter Array (SMA; Ho et al. 2004) observations detected SO peaking between sources A and B (Murillo \& Lai 2013). Even more interesting is the detection of a variable $\mathrm{H}_{2} \mathrm{O}$ maser centered on VLA $1623 \mathrm{~B}$ (Furuya et al. 2003), which was detected between 8 and $13 \mathrm{~km} \mathrm{~s}^{-1}$. Suggestions have been made that VLA $1623 \mathrm{~B}$ could be a background object, such as a galaxy. The velocity shift of the $\mathrm{H}_{2} \mathrm{O}$ maser would suggest a redshift of $3-4 \times 10^{-5}$. Thus, if the maser is associated with VLA $1623 \mathrm{~B}$, then this source is located within our galaxy. VLA $1623 \mathrm{~W}$, separated by $10^{\prime \prime}$ from VLA $1623 \mathrm{~A}$, presents hints of a rotating envelope or disk-like structure, and a slightly different systemic velocity $\left(\sim 1 \mathrm{~km} \mathrm{~s}^{-1}\right)$ from its companions VLA $1623 \mathrm{~A}$ and $\mathrm{B}\left(\sim 3.5 \mathrm{~km} \mathrm{~s}^{-1}\right)$ (Murillo et al. 2013).

In this paper, we present Atacama Large Millimeter/ submillimeter Array (ALMA) Cycle 2 Band 9 dust continuum observations of VLA 1623. These data, combined with previous ALMA Bands 6 and 7 observations, continuum fluxes found in literature, and fluxes from Spitzer and Herschel observations, are used to construct and analyze the spectral energy distribution (SED) of each component in VLA 1623. In 
Table 1. ALMA observations.

\begin{tabular}{lcccccccc}
\hline \hline Project ID & Band & Wavelength & $\begin{array}{c}\text { UV-baseline range } \\
(\mathrm{k} \lambda)\end{array}$ & $\begin{array}{c}\text { Largest scale } \\
\left({ }^{\prime \prime}\right)\end{array}$ & $\begin{array}{c}\text { Field of view } \\
\left({ }^{\prime \prime}\right)\end{array}$ & $\begin{array}{c}\text { Beam } \\
\left({ }^{\prime \prime}\right)\end{array}$ & $\begin{array}{c}\text { P.A. } \\
\left({ }^{\circ}\right)\end{array}$ & $\begin{array}{c}\text { Noise } \\
\left.(\mathrm{mJy} \mathrm{beam})^{-1}\right)\end{array}$ \\
\hline $2011.0 .00902 . \mathrm{S}$ & 6 & $1.3-1.4 \mathrm{~mm}$ & $25 \sim 310$ & 2.5 & 28.1 & $0.79^{\prime \prime} \times 0.54^{\prime \prime}$ & -85.9 & 3.5 \\
$2013.0 .01004 . \mathrm{S}$ & 6 & $1.3-1.4 \mathrm{~mm}$ & $18 \sim 791$ & 2.0 & 28.8 & $0.45^{\prime \prime} \times 0.27^{\prime \prime}$ & 78.4 & 12 \\
$2013.0 .01004 . \mathrm{S}$ & 7 & $828-832 \mu \mathrm{m}$ & $18 \sim 420$ & 3.1 & 17.2 & $0.86^{\prime \prime} \times 0.53^{\prime \prime}$ & -87.1 & 4.2 \\
$2012.1 .00707 . S$ & 9 & $421-429 \mu \mathrm{m}$ & $30 \sim 2280$ & 1.6 & 9.0 & $0.25^{\prime \prime} \times 0.14^{\prime \prime a}$ & -85.1 & $23.9^{a}$ \\
$2012.1 .00707 . S$ & 9 & $421-429 \mu \mathrm{m}$ & $30 \sim 2280$ & 1.6 & 9.0 & $0.17^{\prime \prime} \times 0.1^{\prime \prime} b$ & 85.6 & $14.3^{b}$ \\
\hline
\end{tabular}

Notes. ${ }^{(a)}$ Continuum image generated with natural weighting. ${ }^{(b)}$ Continuum image generated with robust (Briggs) weighting.

addition, newly reduced Spitzer IRS spectra for VLA 1623 are also presented.

\section{Observations}

Table 1 details the ALMA observations presented in this work, including the field of view, angular resolution, and typical noise level.

Cycle 2 observations in Band 9 (Project code: 2012.1.00707.S; PI: D. Salter) have phase center $\alpha_{\mathrm{J} 2000}=16: 26: 26.42 ; \delta_{\mathrm{J} 2000}=$ $-24: 24: 30.0$. However the observations required rephasing, and the phase center was shifted to the position of VLA $1623 \mathrm{~A}$, $\alpha_{\mathrm{J} 2000}=16: 26: 26.39 ; \delta_{\mathrm{J} 2000}=-24: 24: 30.688$, which is consistent with the Cycle 2 Bands 6 and 7 observations (see below). The spectral setup was configured to observe mainly $\mathrm{CO}, \mathrm{HCO}^{+}$, and continuum. J1924-2914 was used for bandpass calibration. Phase calibration was carried out using the check source J17002610 , with a time bin of $24 \mathrm{~s}$, instead of $6 \mathrm{~s}$. Flux calibration was carried out with J1733-130. Ceres was observed, but it was resolved on some baselines, and thus did not provide a reliable flux calibration. Observations toward VLA 1623 did not have any system temperature measurements, so the system temperature of the phase calibrators was used.

Cycle 2 observations in Bands 6 and 7 (Project code: 2013.0.01004; PI: S.-P. Lai) have a phase center of $\alpha_{\mathrm{J} 2000}=16: 26: 26.390 ; \quad \delta_{\mathrm{J} 2000}=-24: 24: 30.688, \quad$ and targeted several molecular lines (e.g., $\mathrm{DCO}^{+}, \mathrm{CO}$ isotopologs, and c- $\mathrm{C}_{3} \mathrm{H}_{2}$ in Band 6, $\mathrm{DCO}^{+}, \mathrm{N}_{2} \mathrm{H}^{+}$, and $\mathrm{H}_{2} \mathrm{D}^{+}$in Band 7) along with continuum. The molecular line observations are detailed in Santangelo et al. (2015) and Murillo et al. (2015, 2018). ALMA Cycle 0 Band 6 observations (Project code: 2011.0.00902.S; PI: N. Murillo) of VLA 1623, which have phase center $\alpha_{\mathrm{J} 2000}=16: 26: 26.419 ; \delta_{\mathrm{J} 2000}=-24: 24: 29.988$ and are slightly offset from the position of VLA $1623 \mathrm{~A}$, were previously reported in Murillo et al. (2013).

The Spitzer IRS spectra are new reductions of serendipitous observations of VLA 1623. The VLA $1623 \mathrm{~W}$ and blended VLA $1623 \mathrm{~A}$ and B components were aligned along the LL slit of the second target in the clustered AOR 12697600 (PID 2) and are visible in the second nod. The basic-calibrated data were downloaded from the Spitzer Heritage Archive and reduced by hand using the AdOpt optimal point source extraction routines from the CASSIS low-resolution pipeline update, version 7 (Lebouteiller et al. 2010) provided in SMART (Higdon et al. 2004). The VLA $1623 \mathrm{~W}$ and blended VLA $1623 \mathrm{~A}$ and B components were fit simulataneously, along with a low-order polynomial sky background, at each wavelength. The resulting spectra are complete in LL $(14-35 \mu \mathrm{m})$ for source $\mathrm{W}$, but the flux of the blended sources A and B is only detected longward of $19 \mu \mathrm{m}$.

\section{Results}

Measured fluxes from ALMA observations are listed in Table 2. VLA 1623 A presents a central bright component surrounded by extended emission in all bands (Fig. 1). In the Band 9 observations, source A does not show further multiplicity, and the clumpiness of the extended dust emission is most likely due to the difficulty of recovering extended structure with high spatial resolution observations. The extended dust emission from Cycle 2 Band 6 data was found to have a radius of $\sim 110 \mathrm{AU}$ (Persson et al. 2016), that is, smaller than that of the Keplerian disk (170 AU at a distance of $137.3 \mathrm{pc})$ traced with $\mathrm{C}^{18} \mathrm{O}$ (Murillo et al. 2013). In contrast, VLA $1623 \mathrm{~B}$ is observed to be compact, almost point-like in all bands. The extended emission in the Band 9 image is most likely not real and a product of the difficulty of recovering structure with high spatial resolution observations. Similarly, VLA $1623 \mathrm{~W}$ is also point-like in Bands 6 and 7, but with a continuum flux 3-5 times weaker than VLA 1623 B (Table 2). Fluxes for each component are measured using a region that is defined by the $3 \sigma$ contour of the continuum emission. For Band 9 continuum, we use the same region used for Band 6. For VLA $1623 \mathrm{~A}$, the extended dust disk component is included in the region used for flux measurement. Measured fluxes are listed in Table 2. VLA 1623 A is brighter than VLA 1623 B by a factor up to 2, although this difference in fluxes might be caused by the inclusion of the dust disk in the flux of VLA $1623 \mathrm{~A}$, and assuming that VLA $1623 \mathrm{~B}$ has no disk. VLA $1623 \mathrm{~W}$ is dimmer than VLA $1623 \mathrm{~A}$ and B by about a factor of 5-12.

\section{Analysis}

In order to construct the SEDs of VLA 1623, the fluxes presented in this work are used together with those found in literature. To constrain the peak of the SEDs, archival Herschel Space Observatory (Pilbratt et al. 2010) PACS photometric maps for Ophiuchus from the Gould Belt Survey (André et al. 2010; Pezzuto et al. 2012) and from the program of PI: P. Ábrahám (Alves de Oliveira et al. 2013) were obtaifrom the Herschel Science Archive (Observation IDs: 134222148 and 1342238816). The maps produced with the JScanmap method were used. The 160 and $100 \mu \mathrm{m}$ maps show the emission toward VLA 1623 to be concentrated mainly on the position of VLA 1623 A and B. A distinct VLA $1623 \mathrm{~W}$ emission is not resolved at either wavelength, most likely due to the resolution of the Herschel observations. At $70 \mu \mathrm{m}$, two distinct continuum emission components are detected in the maps: one centered on VLA 1623 A and B, and the other on VLA $1623 \mathrm{~W}$. Both components show extended emission, most likely arising from their outflow cavities. Because of the nature of the sources, point spread funtion (PSF) photometry is performed on the PACS maps to extract fluxes 
Table 2. SED for each component in VLA1623.

\begin{tabular}{|c|c|c|c|c|c|c|c|c|}
\hline & \multicolumn{2}{|c|}{ VLA $1623 \mathrm{~A}$} & \multicolumn{2}{|c|}{ VLA 1623 B } & \multicolumn{2}{|c|}{ VLA $1623 \mathrm{~W}$} & & \\
\hline RA & \multicolumn{2}{|c|}{$16: 26: 26.390$} & \multicolumn{2}{|c|}{$16: 26: 26.309$} & \multicolumn{2}{|c|}{$16: 26: 25.636$} & & \\
\hline Dec & $-24: 24$ & 30.688 & $-24: 24$ : & 0.588 & $-24: 24:$ & .488 & & \\
\hline $\begin{array}{c}\text { Wavelength } \\
\mu \mathrm{m}\end{array}$ & $\begin{array}{c}S_{\text {int }} \\
\text { mJy }\end{array}$ & $\begin{array}{c}\text { error } \\
\text { mJy }\end{array}$ & $\begin{array}{l}S_{\text {int }} \\
\text { mJy }\end{array}$ & $\begin{array}{l}\text { error } \\
\text { mJy }\end{array}$ & $\begin{array}{l}S_{\text {int }} \\
\text { mJy }\end{array}$ & $\begin{array}{l}\text { error } \\
\text { mJy }\end{array}$ & Ref. & Telescope \\
\hline 3.6 & & & & & 0.74 & 0.12 & 1 & Spitzer/IRAC1 \\
\hline 4.5 & & & & & 2.35 & 0.19 & 1 & Spitzer/IRAC2 \\
\hline 5.8 & & & & & 3.26 & 0.33 & 1 & Spitzer/IRAC3 \\
\hline 8.0 & & & & & 2.60 & 0.26 & 1 & Spitzer/IRAC4 \\
\hline 24 & \multicolumn{4}{|c|}{$40.4 \pm 4.2^{a}$} & 164.26 & 4.54 & 2,1 & Spitzer/MIPS1 \\
\hline $70^{b}$ & \multicolumn{4}{|c|}{$17424.5 \pm 3.2^{a}$} & 5790.8 & 3.88 & 3 & Herschel/PACS \\
\hline $100^{b}$ & \multicolumn{4}{|c|}{$55092.3 \pm 8.7^{a}$} & $10284.9^{c}$ & 8.6 & 3 & Herschel/PACS \\
\hline $160^{b}$ & \multicolumn{4}{|c|}{$81340.6 \pm 10.3^{a}$} & $21457.2^{c}$ & 11.5 & 3 & Herschel/PACS \\
\hline 450 & 3820.0 & 4.2 & 1723.0 & 5.7 & & & 3 & ALMA/Band 9 Natural weighting \\
\hline 450 & 2910.0 & 6.4 & 1653.0 & 6.8 & & & 3 & ALMA/Band 9 Briggs weighting \\
\hline 850 & 1139.5 & 3.4 & 373.0 & 4.6 & 69.0 & 7.0 & 3 & ALMA/Band 7 \\
\hline 1300 & 184.0 & 13.8 & 104.0 & 15.3 & 38.0 & 3.8 & 3 & ALMA/Band 6 Cycle 0 \\
\hline 1300 & 178.0 & 5.1 & 106.0 & 5.3 & 30.0 & 3.0 & 3 & ALMA/Band 6 Cycle 2 \\
\hline 1300 & 152.0 & 8.0 & 106.0 & 8.0 & 25.7 & 8.0 & 4 & SMA \\
\hline 2700 & 25.5 & 6.3 & 25.8 & 3.5 & 3.1 & 1.5 & 5 & BIMA \\
\hline 7000 & 1.2 & 0.2 & 2 & 0.2 & & & 6 & VLA \\
\hline 36000 & 0.13 & 0.02 & 0.4 & 0.02 & 0.2 & 0.02 & 6,7 & VLA \\
\hline 44000 & \multicolumn{2}{|c|}{$<0.2$} & \multicolumn{2}{|c|}{$<0.2$} & & & 6 & MERLIN \\
\hline
\end{tabular}

Notes. ${ }^{(a)}$ Unresolved fluxes. ${ }^{(b)}$ Fluxes obtained with StarFinder PSF photometry using an aperture mask of 11.2", 12.8", and 19.2" for 70, 100, and $160 \mu \mathrm{m}$, respectively. The aperture correction factors are $0.791,0.795$, and 0.789 for 70,100 , and $160 \mu \mathrm{m}$, respectively (Balog et al. 2014). ${ }^{(c)}$ VLA $1623 \mathrm{~W}$ fluxes obtained using the deblend option in StarFinder, since no distinct peak for VLA $1623 \mathrm{~W}$ is observed.

References. (1) Gutermuth et al. (2009); (2) c2d catalog; (3) this work; (4) Murillo \& Lai (2013); (5) Looney et al. (2000); (6) Ward-Thompson et al. (2011); (7) Bontemps \& Andre (1997).

using StarFinder (Diolaiti et al. 2000). The PSF for each wavelength is extracted from the corresponding PACS maps using sources that appear as point sources and have little to no extended emission around them. An aperture mask is applied to the PSF, thus minimizing the extended emission that can be included during photometry. Due to the aperture mask, an aperture correction factor obtained from Balog et al. (2014) is applied to the fluxes. At 160 and $100 \mu \mathrm{m}$, deblending of the detected sources was carried out to obtain fluxes for VLA $1623 \mathrm{~W}$. The method is further detailed in Murillo et al. (2016) and Diolaiti et al. (2000).

Table 2 lists the fluxes and Fig. 2 shows the SED of each component. At wavelengths smaller than $450 \mu \mathrm{m}$, VLA $1623 \mathrm{~A}$ and $\mathrm{B}$ are spatially unresolved. Observations with SMA at $1.3 \mathrm{~mm}$ and BIMA at $2.7 \mathrm{~mm}$ (Looney et al. 2000; Murillo \& Lai 2013) detect all three components. In both datasets, VLA $1623 \mathrm{~A}$ is the brightest of the three sources, while VLA $1623 \mathrm{~B}$ and $\mathrm{W}$ are point-like. The extended emission around VLA $1623 \mathrm{~A}$ is resolved with the SMA (synthesized beam: $0.61^{\prime \prime} \times 0.43^{\prime \prime}, 10^{\circ}$ ) but not in the BIMA observations (synthesized beam: $0.95^{\prime \prime} \times 0.39^{\prime \prime}, 18^{\circ}$ ). Spitzer observations detect VLA $1623 \mathrm{~W}$ in all IRAC and MIPS channels, while unresolved VLA $1623 \mathrm{~A}$ and B are detected only in MIPS1 (Murillo \& Lai 2013), indicating that both sources are deeply embedded. IRS spectra for VLA $1623 \mathrm{~W}$, and unresolved VLA $1623 \mathrm{~A}$ and B are included in the SEDs.

The SEDs are fit using the Scipy method curve_fit ${ }^{1}$ with a single-temperature graybody, and with optical depth $\tau \propto \nu^{\beta}$, where $\beta$ is the dust emissivity index. The fit is carried out in the wavelength range from $7 \mathrm{~mm}$ to $22 \mu \mathrm{m}$. The best fit curves are

\footnotetext{
1 https://www.scipy.org/
}

shown in Fig. 2. The graybody fits are practically the same with or without the Herschel PACS fluxes, which is to be expected since the Herschel fluxes are picking up the envelope material as well. In the submillimeter regime, the SEDs of VLA $1623 \mathrm{~A}$ and $\mathrm{B}$ are very similar, as shown in Fig. 2 with open and filled symbols. For sources $\mathrm{A}$ and $\mathrm{B}$, the unresolved emission at $\lambda<\mu \mathrm{m}$ is fitted in three ways. First, assuming all the infrared emission belongs to one of the sources (Fig. 2 solid red line). Second, the infrared emission is evenly distributed between both sources (Fig. 2 dashed red line). Third, VLA 1623 B only contributes one-tenth of the infrared emission (Fig. 2 dotted red line). The resulting dust temperatures $T_{\text {dust }}$ are similar for all three fits, and for both systems, between 40 and $50 \mathrm{~K}$. However, it is not certain if the emission is evenly distributed at all wavelengths or varies with wavelength. Thus, there are large uncertainties in the SED fit of VLA 1623 A and B. However, the derived dust temperature for VLA $1623 \mathrm{~B}(38-49 \mathrm{~K})$ is larger by an order of magnitude than previously expected ( $T_{\text {dust }}=5 \mathrm{~K}$, Murillo \& Lai 2013). The derived dust temperature for VLA $1623 \mathrm{~W}(66 \mathrm{~K})$ is also higher than previously reported ( $T_{\text {dust }}=41 \mathrm{~K}$, Murillo \& Lai 2013). The higher dust temperature is most likely due to the addition of the IRS spectra to the SED, which was not included in previous studies.

The bolometric luminosity is calculated based on the curve of the fits to the constructed SEDs using the trapezoid method. Considering the three cases used for SED fitting, the bolometric luminosity of VLA $1623 \mathrm{~A}$ is between $\sim 0.4$ and $0.5 L_{\odot}$, while for VLA $1623 \mathrm{~B}$ it is between $\sim 0.2$ and $0.3 L_{\odot}$. It must be stressed that because the SED is unresolved at wavelengths shorter than $450 \mu \mathrm{m}$ for VLA $1623 \mathrm{~A}$ and $\mathrm{B}$, parameters derived from the 


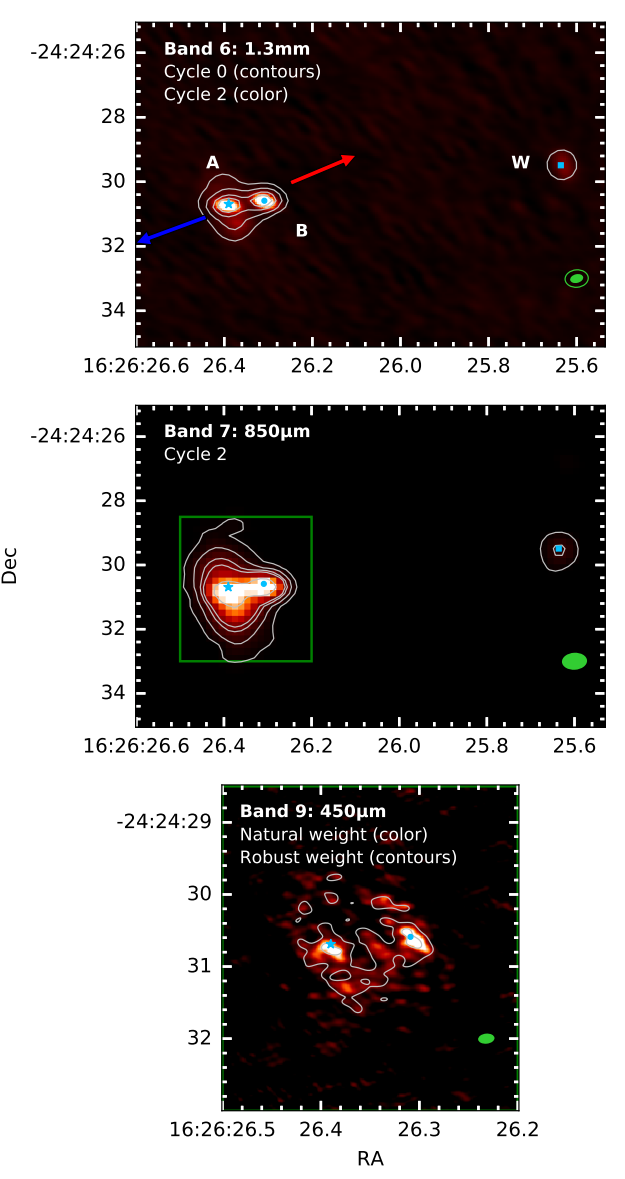

Fig. 1. VLA 1623 observed with ALMA. The phase center for all observations is similar and located around the position of VLA $1623 \mathrm{~A}$. Contours are in steps of 3,8,15,20,50 $\sigma ; \sigma$ is listed in Table 1 . The green ellipses show the beam size of the observations. The green box in the middle panel outlines the area of the Band 9 observations presented in the bottom panel.

SED are uncertain, and these bolometric luminosities are rough estimates. In addition, source A appears brighter since the emission from the dust disk is included. For VLA $1623 \mathrm{~W}$, the derived bolometric luminosity is on the order of $0.13 \pm 0.01 L_{\odot}$.

To calculate the envelope mass, the formula from Jørgensen et al. (2009), which takes into account that brighter sources have somewhat higher dust temperatures, is used and expressed as

$M_{\mathrm{env}}=0.44 M_{\odot}\left(\frac{L_{\mathrm{bol}}}{1 L_{\odot}}\right)^{-0.36}\left(\frac{S_{850 \mu \mathrm{m}}}{1 \mathrm{Jy} \mathrm{beam}^{-1}}\right)^{1.2}\left(\frac{d}{125 \mathrm{pc}}\right)^{1.2}$.

For this calculation, the $850 \mu \mathrm{m}$ fluxes obtained with ALMA Cycle 2 Band 7 observations are used in conjunction with the bolometric luminosities derived from the SEDs and described above. This method yields a mass of $\sim 0.8$ and $0.2 M_{\odot}$ for VLA $1623 \mathrm{~A}$ and B, respectively, for a distance of $137.3 \mathrm{pc}$. For VLA $1623 \mathrm{~W}$, the mass is $0.04 \pm 0.005 M_{\odot}$. If using the previous distance to VLA 1623 of $120 \mathrm{pc}$ (Loinard et al. 2008), the masses are decreased by $15 \%$. Several caveats need to be highlighted here. The $850 \mu \mathrm{m}$ flux from ALMA Cycle 2 observations only recovers emission from the disk and inner envelope (up to $3^{\prime \prime}$, $\sim 400 \mathrm{AU})$, but not from the large scale gas (>400 AU). Thus the mass estimate reported in this work is not representative of the whole envelope mass. Furthermore, if VLA 1623 B is observed through the envelope of $\mathrm{A}$, then the mass estimate for $\mathrm{B}$ is in fact not indicative of its actual mass.
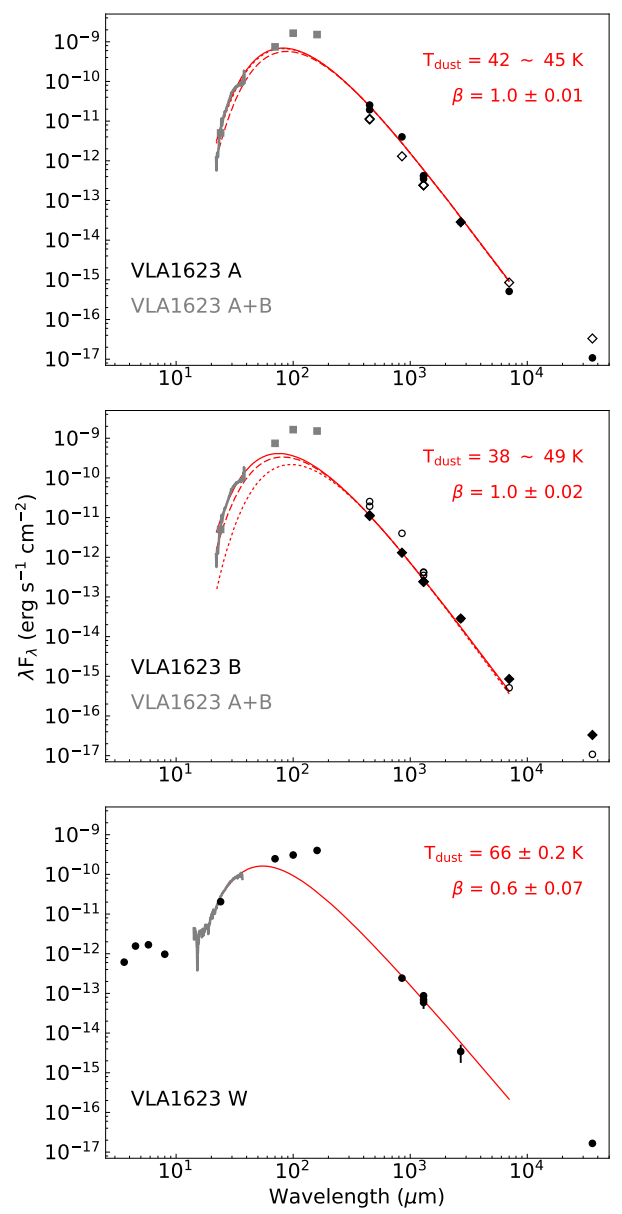

Fig. 2. Spectral energy distribution of VLA 1623 including the Spitzer IRS spectra. The red curves show the best fit graybody to each SED. The dust temperature $T_{\text {dust }}$ and dust emissivity index $\beta$ are indicated in each panel. For VLA 1623 A (top) and B (middle), the dashed curves show the best fit assuming that the emission at $\lambda<450 \mu \mathrm{m}$ is evenly distributed among the two sources. The dotted curve assumes VLA $1623 \mathrm{~B}$ only contributes one-tenth of the total emission for $\lambda<450 \mu \mathrm{m}$. The open symbols in the top and middle panel are the fluxes for VLA $1623 \mathrm{~B}$ and VLA 1623 A, respectively, and are included for ease of comparison.

\section{Summary}

In this paper we present new ALMA Band 9 continuum data toward VLA 1623, resolving the components A and B. The PSF photometry of Herschel PACS maps provides unresolved fluxes between 70 and $160 \mu \mathrm{m}$, which trace the peak of the SED. The updated SEDs show that VLA 1623 B does not peak at $1000 \mu \mathrm{m}$, as suggested by Murillo \& Lai (2013) based on SMA $850 \mu \mathrm{m}$ observations, but might instead peak at around $100 \mu \mathrm{m}$, similar to VLA $1623 \mathrm{~A}$. The best fit using a graybody indicates that the dust temperature of both sources is in the $40-50 \mathrm{~K}$ range. There are large uncertainties, however, because of the unresolved fluxes of VLA 1623 A and B at wavelengths shorter than $450 \mu \mathrm{m}$, and thus the derived dust temperature should be considered with caution. With these results, the previous assumption that the continuum flux at shorter wavelengths is dominated by VLA $1623 \mathrm{~A}$ is not well based, and instead VLA 1623 B could be the dominant source in the infrared regime.

Deriving source parameters or evolutionary stage from unresolved SEDs is nontrivial, and can lead to under- or overestimation of the parameters (see, e.g., Murillo et al. 2016). Keeping this caveat in mind, the bolometric luminosity derived for 
VLA $1623 \mathrm{~A}$ and B from the SEDs presented in this work, together with the rough estimate of the disk and inner envelope mass on scales up to $400 \mathrm{AU}$, provide constraints on the source parameters for each component, albeit with large uncertainties. The bolometric luminosity for VLA 1623 A and B varies by a factor of $\sim 2$, and the mass of the disk plus inner envelope varies by a factor of $\sim 4$ since it is dependent on the luminosity and $850 \mu \mathrm{m}$ flux. All parameters derived from the SED indicate that both sources are Class 0 . In the scenario in which source B would dominate the infrared emission, its bolometric luminosity would rise and the mass would decrease. For VLA $1623 \mathrm{~W}$, the luminosity is lower by a factor of $\sim 4$ compared to source $A$, while the envelope mass is lower by a factor of 20 compared to that of source A, which is consistent with its classification as a Class I source.

Along with separating the fluxes of components in a multiple protostellar system, the variability of the sources must also be taken into account when understanding the nature of these systems. Recent studies show variation in the brightness of protostellar systems, possibly owing to episodic accretion processes (e.g., Herczeg et al. 2017; Johnstone et al. 2018). For the case of VLA 1623, however, all three components have not shown significant variations in brightness in the submillimeter range. This can be noted in the $1.3 \mathrm{~mm}$ fluxes (Table 2), which have been obtained with the SMA (2007 and 2009) and ALMA in Cycle 0 (2011) and Cycle 2 (2013). The relative similarity of the fluxes at $1.3 \mathrm{~mm}$ suggests that there have been no large variations in brightness. There could be variations on the order of $10 \%$ at $1.3 \mathrm{~mm}$, but given the uncertainties of the flux calibration, such variations would not be detected. In addition to variability in brightness, chemical signatures in the envelope gas can be used to probe episodic accretion (Visser et al. 2015; Jørgensen et al. 2015; Frimann et al. 2017; Hsieh et al. 2018). Considering the characterized gas structure of VLA 1623 (Murillo et al. 2013, 2015,2018 ), no chemical signatures of luminosity variations toward the components of VLA 1623 are detected.

The presented results contribute to the puzzle of VLA $1623 \mathrm{~B}$. The derived dust temperature from the SED fitting $(40-50 \mathrm{~K})$ suggests there should be warm gas emission from this source. However, no circumstellar gas emission in either cold or warm molecules (Murillo et al. 2013, 2015, 2018) has been detected to be associated with VLA $1623 \mathrm{~B}$. In contrast, VLA $1623 \mathrm{~B}$ drives a fast and collimated jet and outflow (Santangelo et al. 2015), and presents a variable $\mathrm{H}_{2} \mathrm{O}$ maser (Furuya et al. 2003). The lack of detected circumstellar gas emission around VLA $1623 \mathrm{~B}$ is difficult to explain. One possibility could be gas stripping due to interaction with the outflow from VLA 1623 A or VLA $1623 \mathrm{~W}$ (e.g., B1-b: Hirano \& Liu 2014), but this would not explain why the dust was not stripped or presence of the outflow. Another explanation would be that VLA $1623 \mathrm{~B}$ is more evolved than VLA $1623 \mathrm{~A}$. However this would not fully explain the complete lack of gas in submillimeter when VLA $1623 \mathrm{~W}$ presents circumstellar gas (Murillo et al. 2013). A final possibility would be that VLA $1623 \mathrm{~B}$ is being obscured, either by the envelope of VLA 1623 A or circumstellar matter with high optical depth. This would also introduce further uncertainties to the calculated envelope mass. Observations with subarcsecond resolution in the infrared are required to determine the nature of this object, for which the James Webb Space Telescope will be ideal.

Acknowledgements. This paper made use of the following ALMA data ADS/JAO.ALMA 2011.0.00902.S, 2012.1.00707.S, and 2013.1.01004.S. ALMA is a partnership of ESO (representing its member states), NSF (USA), and NINS (Japan), together with NRC (Canada) and NSC and ASIAA (Taiwan), in cooperation with the Republic of Chile. The Joint ALMA Observatory is operated by ESO, AUI/NRAO, and NAOJ. The authors acknowledge assistance from Allegro, the European ALMA Regional Center node in the Netherlands. Herschel is an ESA space observatory with science instruments provided by European-led Principal Investigator consortia and with important participation from NASA. PACS has been developed by a consortium of institutes led by MPE (Germany) and including UVIE (Austria); KU Leuven, CSL, IMEC (Belgium); CEA, LAM (France); MPIA (Germany); INAF-IFSI/OAA/OAP/OAT, LENS, SISSA (Italy); IAC (Spain). This development has been supported by the funding agencies BMVIT (Austria), ESA-PRODEX (Belgium), CEA/CNES (France), DLR (Germany), ASI/INAF (Italy), and CICYT/MCYT (Spain). This work is based in part on observations made with the Spitzer Space Telescope, which is operated by the Jet Propulsion Laboratory, California Institute of Technology under a contract with NASA. NMM and DH are supported by the European Union A-ERC grant 291141 CHEMPLAN, by the Netherlands Research School for Astronomy (NOVA), by a Royal Netherlands Academy of Arts and Sciences (KNAW) professor prize. MM receives funding through a Marie Skłodowska-Curie action in the Horizon 2020 program. SPL acknowledges support from the Ministry of Science and Technology of Taiwan with Grant MOST 106-2119-M-007-021-MY3. MRH is support by grant 614.001.352 from the Netherlands Organisation for Scientific Research (NWO). The authors wish to thank Doug Johnstone for the helpful and insightful comments that greatly improved this work.

\section{References}

Alves de Oliveira, C., Ábrahám, P., Marton, G., et al. 2013, A\&A, 559, A126 André, P., Martin-Pintado, J., Despois, D., \& Montmerle, T. 1990, A\&A, 236, 180

André, P., Ward-Thompson, D., \& Barsony, M. 1993, ApJ, 406, 122

André, P., Men'shchikov, A., Bontemps, S., et al. 2010, A\&A, 518, L102

Balog, Z., Müller, T., Nielbock, M., et al. 2014, Exp. Astron., 37, 129

Bontemps, S., \& Andre, P. 1997, in Herbig-Haro Flows and the Birth of Stars, eds. B. Reipurth, \& C. Bertout, IAU Symp., 182, 63

Caratti o Garatti, A., Giannini, T., Nisini, B., \& Lorenzetti, D. 2006, A\&A, 449, 1077

Dent, W. R. F., Matthews, H. E., \& Walther, D. M. 1995, MNRAS, 277, 193

Diolaiti, E., Bendinelli, O., Bonaccini, D., et al. 2000, in Adaptive Optical

Systems Technology, ed. P. L. Wizinowich, SPIE Conf. Ser., 4007, 879-888

Frimann, S., Jørgensen, J. K., Dunham, M. M., et al. 2017, A\&A, 602, A120

Furuya, R. S., Kitamura, Y., Wootten, A., Claussen, M. J., \& Kawabe, R. 2003, ApJS, 144, 71

Gutermuth, R. A., Megeath, S. T., Myers, P. C., et al. 2009, ApJS, 184, 18

Herczeg, G. J., Johnstone, D., Mairs, S., et al. 2017, ApJ, 849, 43

Higdon, S. J. U., Devost, D., Higdon, J. L., et al. 2004, PASP, 116, 975

Hirano, N., \& Liu, F.-c. 2014, ApJ, 789, 50

Ho, P. T. P., Moran, J. M., \& Lo, K. Y. 2004, ApJ, 616, L1

Hsieh, T.-H., Murillo, N. M., Belloche, A., et al. 2018, ApJ, 854, 15

Johnstone, D., Herczeg, G. J., Mairs, S., et al. 2018, ApJ, 854, 31

Jørgensen, J. K., van Dishoeck, E. F., Visser, R., et al. 2009, A\&A, 507, 861

Jørgensen, J. K., Visser, R., Williams, J. P., \& Bergin, E. A. 2015, A\&A, 579, A23

Lebouteiller, V., Bernard-Salas, J., Sloan, G. C., \& Barry, D. J. 2010, PASP, 122, 231

Loinard, L., Torres, R. M., Mioduszewski, A. J., \& Rodríguez, L. F. 2008, ApJ, 675, L29

Looney, L. W., Mundy, L. G., \& Welch, W. J. 2000, ApJ, 529, 477

Murillo, N. M., \& Lai, S.-P. 2013, ApJ, 764, L15

Murillo, N. M., Lai, S.-P., Bruderer, S., Harsono, D., \& van Dishoeck, E. F. 2013, A\&A, 560, A103

Murillo, N. M., Bruderer, S., van Dishoeck, E. F., et al. 2015, A\&A, 579, A114

Murillo, N. M., van Dishoeck, E. F., Tobin, J. J., \& Fedele, D. 2016, A\&A, 592, A56

Murillo, N. M., van Dishoeck, E. F., van der Wiel, M. H. D., et al. 2018, A\&A, in press, DOI: $10.1051 / 0004-6361 / 201731724$

Ortiz-León, G. N., Loinard, L., Kounkel, M. A., et al. 2017, ApJ, 834, 141

Persson, M. V., Harsono, D., Tobin, J. J., et al. 2016, A\&A, 590, A33

Pezzuto, S., Elia, D., Schisano, E., et al. 2012, A\&A, 547, A54

Pilbratt, G. L., Riedinger, J. R., Passvogel, T., et al. 2010, A\&A, 518, L1

Santangelo, G., Murillo, N. M., Nisini, B., et al. 2015, A\&A, 581, A91

Visser, R., Bergin, E. A., \& Jørgensen, J. K. 2015, A\&A, 577, A102

Ward-Thompson, D., Kirk, J. M., Greaves, J. S., \& André, P. 2011, MNRAS, 415,2812

Yu, T., \& Chernin, L. M. 1997, ApJ, 479, L63 